\title{
ORIENTAÇÃO EXTERIOR DE UM PAR ESTEREOSCÓPICO IKONOS II COM BASE NO MODELO NÃO-RIGOROSO DLT
}

\author{
Exterior orientation of a stereo pair of IKONOS II images based on DLT non-rigorous
} model.

\author{
SYDNEY DE OLIVEIRA DIAS ${ }^{1}$ \\ EDSON APARECIDO MITISHITA ${ }^{2}$ \\ QUINTINO DALMOLIN ${ }^{2}$ \\ ${ }^{1}$ Instituto Federal de Educação, Ciência e Tecnologia da Paraíba - IFPB \\ Curso Superior de Tecnologia em Geoprocessamento \\ Av. $1^{\circ}$ de Maio, 720 , Jaguaribe \\ CEP 58015-430 - João Pessoa - PB - Brasil \\ sydney.dias@ifpb.edu.br \\ ${ }^{2}$ Universidade Federal do Paraná - UFPR \\ Curso de Pós-Graduação em Ciências Geodésicas - CPGCG \\ Caixa Postal 515 - Centro Politécnico - Jardim das Américas \\ CEP 12201-970 - Curitiba - PR - Brasil \\ \{mitishita; qdalmolin\}@ufpr.br
}

\begin{abstract}
RESUMO
O crescente avanço tecnológico ocorrido nos últimos anos, tanto da área da Informática, Fotogrametria e também Sensoriamento Remoto, vem contribuindo significativamente para diversas pesquisas sobre extração de informações tridimensionais a partir de imagens de alta resolução. No presente trabalho, uma solução utilizando modelagem não rigorosa baseada na transformação matemática DLT (Direct Linear Transformation) foi utilizada para a orientação exterior de um par estereoscópico de imagens Ikonos II e determinação de coordenadas tridimensionais por intersecção fotogramétrica. O objetivo principal foi o de avaliar o desempenho do modelo na aplicação, como também a obtenção de melhor configuração de pontos de apoio necessários na orientação exterior para fins da determinação de coordenadas tridimensionais por interseção espacial. Os experimentos realizados permitiram recomendar a configuração adequada para a distribuição dos pontos de apoio, onde a exatidão planimétrica foi equivalente a precisão teórica tolerável igual a um pixel de erro nas observações, enquanto a exatidão altimétrica ficou próxima de 1,5 pixels.
\end{abstract}


Palavras-chave: Orientação Exterior; DLT; Interseção Fotogramétrica; Georreferenciamento Indireto; Configuração de Pontos de Apoio.

\section{ABSTRACT}

The technology improvement which has occurred in areas such as Computer Sciences, Photogrammetry and Remote Sensing has contributed significantly to many researches in $3 \mathrm{D}$ information extraction from high resolution images. The present paper shows a solution to perform the exterior orientation of a stereo pair of Ikonos II images and photogrammetric intersection, using a non rigorous model based on the mathematical model DLT (Direct Linear Transformation). The main research goals is the evaluation of the model performance for this kind of application associated with the best layout of control points to perform the indirect georeferecing of the Ikonos stereo pair to compute 3D coordinates via photogrammetric intersection. From the performed experiments, the best layout of control points was obtained. Using this control point configuration, the experiment yielded horizontal accuracy close to one pixel while vertical accuracy was close to 1.5 pixels.

Keywords: Exterior Orientation; Photogrammetric Intersection; Indirect Georeferencing; DLT; Control Points Layout.

\section{INTRODUÇÃO}

O Sensoriamento Remoto vem contribuindo cada vez mais em diversos trabalhos que envolvem a obtenção de informações espaciais da superfície terrestre referenciadas a um sistema geodésico ou cartográfico.

Segundo Amato et al. (2004), a disponibilidade de imagens de alta resolução espacial em conjunto com Modelos Digitais do Terreno (MTD's) pode ser considerada como sendo uma alternativa aos levantamentos fotogramétricos convencionais na geração de produtos de escala média com exatidão geométrica compatível com mapas na escala entre 1:10.000 e 1:5.000.

Figura 1 - Obtenção de imagem com sensor linear. Fonte: Wolf (2004).

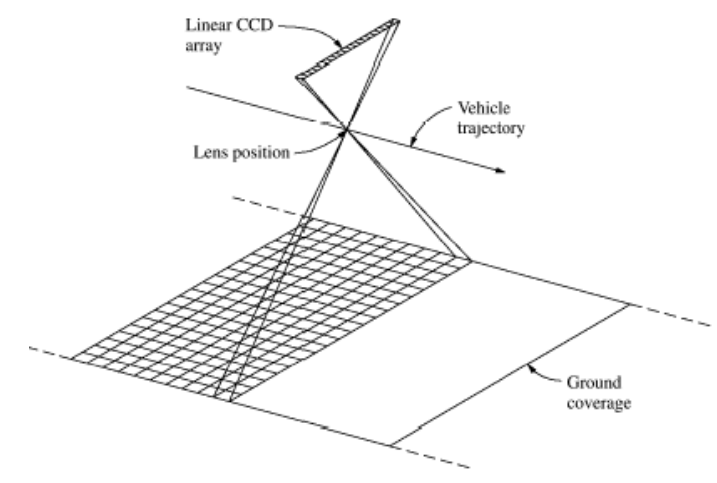

Bol. Ciênc. Geod., sec. Artigos, Curitiba, v. 18, nº 1, p.40-62, jan-mar, 2012. 
A Figura 1 mostra o esquema de obtenção de imagens orbitais que operam com sensores do tipo pushbroom, onde se tem um conjunto de sensores CCD's arranjados linearmente a bordo do satélite. As informações da superfície terrestre são obtidas a partir de uma varredura linear (unidimensional) no sentido do deslocamento da plataforma, diferentemente do que ocorre no sensor de quadro (full-frame sensor), que registra a cena completa num mesmo instante.

Além do IKONOS II, satélites como SPOT, Quickbird, Orbview, J-ERS, ALOS, EROS, MIR, IRS e CBERS utilizam sensores do tipo pushbroom para capturar imagens pancromáticas e multiespectrais com resoluções geométricas que variam de alguns centímetros a centenas de metros. A correção geométrica, ou referenciamento geodésico dessas imagens pode ser realizado empregando-se modelos rigorosos ou aproximados (FRASER et. al 2002). A obtenção de informações sobre os parâmetros físicos do sensor, segundo Pedro (2005), pode ser considerada como sendo um inconveniente na utilização de modelos rigorosos, pois dependendo do tipo de imagem, algumas das informações necessárias não são disponibilizadas pelas empresas que comercializam as imagens.

Um conjunto de pontos geometricamente distribuídos na imagem ou modelo estereoscópico pode ser considerado como sendo um pré-requisito nas metodologias conhecidas para o georreferenciamento indireto de uma imagem ou par estereoscópico com a utilização de modelos não-rigorosos. Neste sentido, a definição de detalhes pontuais perfeitamente visíveis nas imagens e que possuam características que habilitam a obtenção de precisões no levantamento de campo, pode ser admitida como sendo uma etapa imprescindível de trabalho e fundamental para o aumento de exatidão no referenciamento geodésico requerido.

O processo de orientação de um par estereoscópico proveniente de imagens orbitais para fins de intersecção espacial ou fotogramétrica segue os mesmos princípios de reconstrução tridimensional da Fotogrametria. Em aplicações não rigorosas, o modelo fundamental de colinearidade é substituído por modelos matemáticos aproximados, tais como a Transformação Linear Direta - DLT, Projeção Paralela Afim - APM, Afim 3D, Modelo de Razão de Polinômios - RPC. Para a orientação exterior é necessário ter um conjunto de pontos homólogos com coordenadas conhecidas nos sistemas do espaço objeto e do espaço imagem e de um modelo matemático que as relacione. Com base nessas informações, os parâmetros do modelo matemático são determinados via ajustamento por Mínimos Quadrados. Tendo-se o modelo ajustado, a interseção espacial é realizada com a resolução de um sistema com quatro equações e três incógnitas, provenientes das medidas de pontos homólogos realizadas no par estereoscópico.

Visando apresentar uma contribuição neste segmento, realizaram-se pesquisas correlacionadas com a orientação exterior de um par estereoscópico de imagens de alta resolução IKONOS II com base na transformação matemática DLT, tendo como principal objetivo a análise da adequação de utilização do modelo nessa aplicação e obtenção de configuração adequada de pontos de apoio para realização do processo. 
Ou seja, os experimentos foram realizados visando obter configurações de pontos de apoio, em quantidade e distribuição espacial, com o objetivo de orientar o par de imagens para a realização da intersecção espacial com nível de precisões planimétrica e altimétrica equivalente a um erro de um pixel nas observações de imagem.

\section{TRABALHOS RELACIONADOS}

A determinação da transformação matemática (ou modelo matemático) para o relacionamento geométrico entre o espaço imagem e o espaço objeto pode ser considerada como sendo a base dos trabalhos empregados no processo da correção geométrica de imagem orbital e também fundamental quando se deseja determinar coordenadas tridimensionais a partir de pares estereoscópicos.

Pesquisas de orientação de um par estereoscópico para fins de interseção espacial com a utilização do modelo rigoroso podem ser vistas em Fritsch e Stallmann (2000) e Kim e Dowman (2006). Nessa abordagem, para cada linha da imagem existirá um conjunto de parâmetros de orientação exterior. Já a respeito dos parâmetros de orientação interior, como distância focal, localização do ponto principal, coeficientes de distorção e outros parâmetros diretamente relacionados com a concepção física do sensor, são os mesmos para toda imagem.

Procedimentos aproximados são largamente empregados nesta aplicação. Uma alternativa muito utilizada é a utilização do modelo polinomial racional (RFM Rational Function Model). No trabalho de Jacobsen (2007) são apresentados dois principais aspectos a serem considerados para a determinação dos parâmetros de transformação do modelo RMF (Rational Model Function). O primeiro considera a determinação dos parâmetros independentemente do terreno - o que torna imprescindível o conhecimento do modelo físico do sensor. Já o segundo aspecto, trata da determinação dos parâmetros de maneira dependente do terreno, onde são necessários pontos de apoio para determinação dos coeficientes do modelo RPC. Grodecki (2001) realizou experimentos com esta abordagem para a correção geométrica de imagens. Os resultados finais de exatidões obtidas com os RPC foram comparados com resultados utilizando-se modelo físico e a diferença entre eles não foi superior a 0,04 pixel e a diferença entre o RMS das duas abordagens ficou com valores próximos de 0,01 pixel. Os experimentos de Silva e Vergara (2005) na geração de ortoimagens com a utilização do produto Geo Ortho Kit, mostrou-se viável, desde que se tenham disponível um MDT da região. A utilização de alguns pontos de controle serviria para, opcionalmente, melhorar a qualidade da ortoimagem.

Yamakawa e Fraser (2004) apresentam os procedimentos para reconstrução 3D em imagens de alta resolução utilizando-se da projeção Afim Paralela. Evidenciam que a quantidade mínima de pontos de apoio para determinação dos parâmetros de transformação como sendo uma das principais vantagens na utilização do modelo. Por outro lado, de acordo com os autores, a metodologia apresenta limitações, pelo fato do modelo não considerar de forma individual a 
projeção central para cada linha imageada, como também a de não parametrizar os diferentes intervalos de tempo de aquisição de cada linha. Apesar dessas limitações, exatidões próximas do subpixel foram alcançadas com imagens IKONOS e Quickbird.

Pedro (2005) realizou pesquisas de ortorretificação de imagens Ikonos e Quickbird empregando modelos APM e RFM. As exatidões planimétricas obtidas com a APM foram melhores que as obtidas com o RFM que utilizou como base apenas os coeficientes do polinômio (RPC's). Nesta aplicação, o modelo APM foi ajustado com a utilização de pontos de apoio obtidos a partir de uma base cartográfica digital (escala 1:2000). As exatidões planimétricas obtidas nos experimentos realizados com o modelo APM ficaram na ordem de 0,7 a 1,0 m.

El-Manadili e Novak (1996) utilizaram a DLT em modelagem geométrica de imagens SPOT. Savopol e Armenakis (1998) aplicaram o modelo em imagens IRS$1 \mathrm{C}$ e obtiveram resultados planimétricos com acurácia de um pixel. Galindo (2008) realizou experimentos com modelo polinomial e DLT aplicado em estereoimagens do satélite CBERS, tendo como resultados, erro planimétrico na ordem de 1,5 pixel e erro altimétrico superior a 4 pixel. Jacobsen (2007) também efetuou experimentos com a DLT em imagens orbitais IKONOS II, chegando a resultados planimétricos da ordem de 0,5 pixels e altimétrico de 1,0 pixel. Fraser et. al (2002) utilizaram estereoimagens IKONOS para extração de informações tridimensionais. Os experimentos realizados na reconstrução de edificações apresentaram exatidões planimétricas da ordem de 0,5 pixels e altimétricas da ordem de 0,7 pixels. Dal Poz e Scalco (2006) utilizaram modelagem matemática DLT para orientação de imagens CBERS utilizando rodovias como controle de campo e os resultados obtidos permitiram o registro de rodovias (verificação) com exatidão no nível do pixel, ou melhor.

\subsection{Orientação Exterior e Intersecção Espacial com a DLT}

Baseada no princípio da equação da colinearidade com a projeção perspectiva, a DLT foi inicialmente introduzida por Abdel-Aziz e Karara (1971) com objetivo de relacionar diretamente os espaços imagem e objeto sem a necessidade de conhecimento dos parâmetros de orientação interior levando-se em conta o uso de câmaras fotográficas de baixo custo (não calibradas).

O modelo matemático DLT tem como objetivo de estabelecer a relação entre o sistema de coordenadas do espaço imagem e o sistema de coordenadas do espaço objeto de forma semelhante à equação da colinearidade.

Jubanski e Mitishita (2004) citam que, tanto a DLT, como a equação da colinearidade, são casos particulares de modelos polinomiais. Este modelo, por relacionar diretamente as coordenadas dos dois sistemas (imagem e objeto) torna-se bastante simples. Segundo Mitishita et al (2003), a DLT pode ser considerada como sendo uma transformação aproximada da colinearidade, possuindo facilidades no relacionamento direto das coordenadas do sistema de imagem (dada em pixel) com coordenadas geodésicas (X,Y e Z), apresentada como:

Bol. Ciênc. Geod., sec. Artigos, Curitiba, v. 18, no 1, p.40-62, jan-mar, 2012. 


$$
\begin{gathered}
x=\frac{L_{1} X+L_{2} Y+L_{3} Z+L_{4}}{L_{9} X+L_{10} Y+L_{11} Z+1} \\
y=\frac{L_{5} X+L_{6} Y+L_{7} Z+L_{8}}{L_{9} X+L_{10} Y+L_{11} Z+1}
\end{gathered}
$$

onde:

$(\mathrm{x}, \mathrm{y})$ : Coordenadas planas no referencial de imagem (dadas em pixel);

( $\mathrm{X}, \mathrm{Y}$ e Z): Coordenadas 3D no referencial geodésico local;

$\left(\mathrm{L}_{1}, \mathrm{~L}_{2}, \mathrm{~L}_{3}, \ldots, \mathrm{L}_{11}\right)$ : Coeficientes do modelo DLT.

Aproximando-se a forma de aquisição de um par de imagens de alta resolução IKONOS II como sendo obtido na forma de um sensor de quadro, a orientação exterior pode ser realizada com a utilização do modelo matemático da DLT. Como este modelo possui um total de 11 parâmetros (incógnitas), um conjunto mínimo de seis pontos de apoio, com suas coordenadas de imagem e de terreno conhecidas, resultam em 12 equações que possibilitam a solução do problema via ajustamento por mínimos quadrados.

A partir dos parâmetros dos modelos DLT das imagens do par estereoscópico, obtidos no ajustamento de feixes perspectivos e das coordenadas no referencial de imagem de pontos homólogos é possível, com a intersecção espacial, determinar suas coordenadas tridimensionais no referencial geodésico. Um sistema de quatro equações para três incógnitas (coordenadas 3D de terreno) é solucionado pelo Método dos Mínimos Quadrados (MMQ). Maiores informações sobre o ajustamento envolvido no processo de orientação exterior de um modelo fotogramétrico podem ser encontradas em Lugnani (1987) e Merchant (1979).

\section{MATERIAIS E MÉTODOS}

\section{1 Área de Estudo}

A área de estudo localiza-se na região metropolitana de Curitiba-PR, mais precisamente no município de Araucária, Figura 2.

O par estereoscópico utilizado na presente pesquisa são imagens que possuem correção geométrica padrão (Standard Geometrically Corrected), reamostradas para um mapa na projeção cartográfica UTM, com altitude média de referência no valor de $907,967 \mathrm{~m}$ referenciadas ao datum WGS-84 e recortadas nas dimensões aproximadas descritas na Tabela 1. O nome do produto, segundo a empresa Geoeye, é o Reference Stereo, com precisão nominal planimétrica e altimétrica de 15 e 22 metros, respectivamente. 
Figura 2 - Área de estudo.

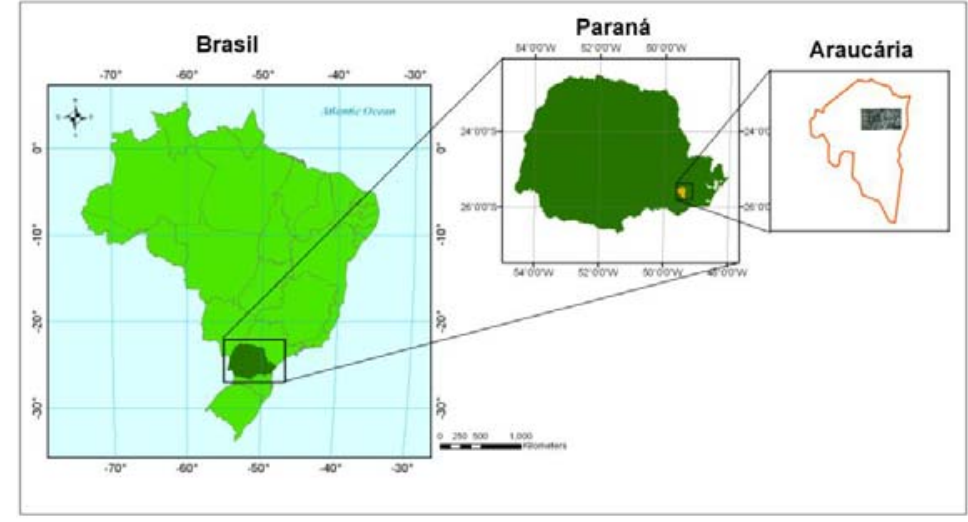

Tabela 1 - Informações das imagens IKONOS utilizadas.

\begin{tabular}{c|c|c}
\hline & $156631-000$ & $156631-001$ \\
\hline & $\begin{array}{c}2004-11-2313: 42 \\
\text { GMT }\end{array}$ & $\begin{array}{c}2004-11-2313: 41 \\
\text { GMT }\end{array}$ \\
\hline DATA/HORA & & \\
AZIMUTE DO & $239^{\circ} 21^{\prime} 57,96^{\prime \prime}$ & $357^{\circ} 07^{\prime} 23,52^{\prime \prime}$ \\
SENSOR & $80^{\circ} 45^{\prime} 09,504^{\prime \prime}$ & $65^{\circ} 08^{\prime} 28,932^{\prime \prime}$ \\
\hline ELEVAÇÃO DO & $79^{\circ} 35^{\prime} 58,92^{\prime \prime}$ & $79^{\circ} 47^{\prime} 0,06^{\prime \prime}$ \\
\hline SENSOR & $70^{\circ} 25^{\prime} 19,38^{\prime \prime}$ & $70^{\circ} 14^{\prime} 12,552^{\prime \prime}$ \\
\hline AZIMUTE SOLAR & $10164 \times 5104$ & $10164 \times 5104$ \\
\hline ELEVAÇÃO SOLAR & &
\end{tabular}

\subsection{Obtenção das Coordenadas de Campo e de Imagem}

Para a obtenção das coordenadas dos pontos de apoio e de verificação utilizadas no presente trabalho, optou-se por um levantamento em campo utilizando técnicas de levantamento GPS em tempo real - Real Time Kinematic (RTK).

A obtenção das coordenadas dos pontos de apoio no referencial de imagem (dada em pixel) e dos pontos de verificação foi efetuada com auxílio de rotina desenvolvida com o programa Matlab onde foi possível medir manualmente com aproximação do subpixel. Foi levantado um total de 50 pontos de campo com GPS, sendo 25 pontos de apoio e 20 de verificação. Foram escolhidos, manualmente, detalhes pontuais com fácil identificação, fácil acessibilidade para o levantamento de campo e de homogênea distribuição por toda a região do par estereoscópico, conforme é mostrado na Figura 3. 
Figura 3 - Configuração dos pontos.

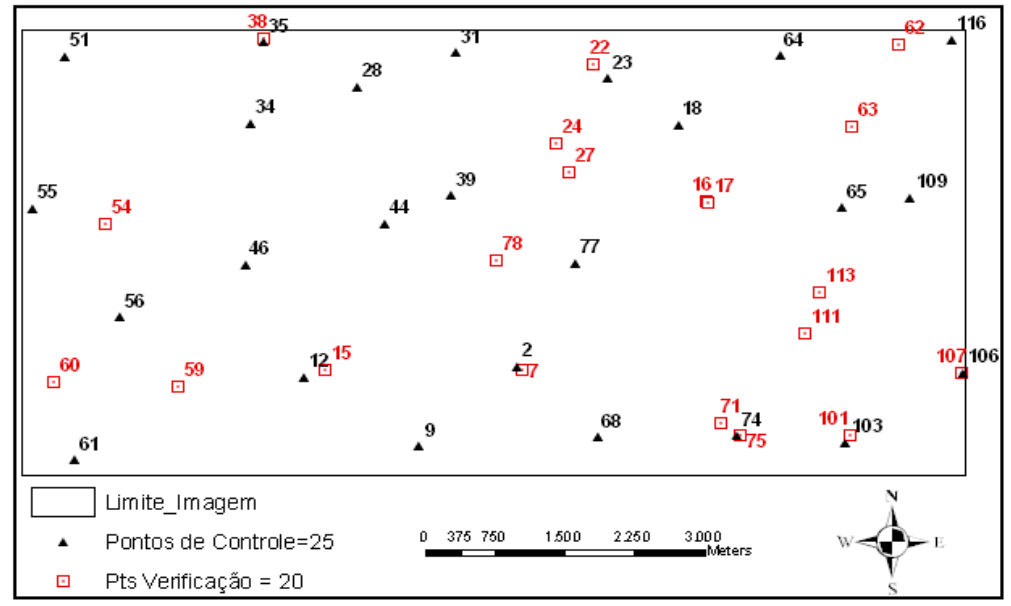

\subsection{Controle de Qualidade de Produtos Cartográficos}

Conforme descrito por Merchant (1982) o processo de avaliação posicional de produtos cartográficos pode ser realizado a partir de duas fases distintas. A primeira consiste de uma análise estatística para verificar a existência de tendência, com base na distribuição " $t$ " de student ou na distribuição normal (NOGUEIRA, 2003). Na segunda fase a exatidão das informações obtidas é efetuada com base na análise estatística do Qui-Quadrado.

De acordo com Galo e Camargo (1994), a exatidão de uma determinada carta é baseada na análise estatística das discrepâncias entre as coordenadas (tri ou bidimensionais) observadas na carta $\left(X_{i}\right)$ e as referidas coordenadas de referência $\left(X_{i}^{r}\right)$, calculadas para cada ponto "i” e dadas por:

$$
\Delta X_{i}=X_{i}-X_{i}^{r}
$$

Os valores da média e do desvio padrão das discrepâncias são calculados por:

$$
\begin{gathered}
\Delta \bar{X}=\frac{1}{n} \cdot \sum_{i=1}^{n} \Delta X_{i} \\
S_{\Delta X}=\sqrt{\frac{1}{n-1} \cdot \sum_{i=1}^{n}\left(\Delta X_{i}-\Delta \bar{X}^{2}\right)}
\end{gathered}
$$

sendo $\mathrm{n}$ igual ao tamanho da amostra. 
As hipóteses formuladas para a verificação da existência de tendência são apresentadas da seguinte forma:

$\mathrm{H}_{0}: \Delta \bar{X}=0$, contra

$\mathrm{H}_{1}: \Delta \bar{X} \neq 0$

Conforme descrito por Nogueira (2003), o teste estatístico com base na distribuição " $t$ " de student é indicado quando o desvio padrão populacional é desconhecido. Entretanto, quando o objetivo é realizar controle de qualidade de produtos cartográficos, em território nacional, torna-se importante levar em consideração a utilização de documentos que estabelecem normas reguladoras para tais atividades.

No Brasil, as Normas Técnicas da Cartografia Nacional são estabelecidas pelos artigos $8^{\circ}$ e $9^{\circ}$ do Decreto Lei 89.917 de 20 de junho de 1984. De acordo com esse decreto a exatidão de produtos cartográficos deve obedecer ao Padrão de Exatidão Cartográfica - PEC, podendo ser classificada nas classes $\mathrm{A}, \mathrm{B}$ ou $\mathrm{C}$, conforme se pode observar no Quadro 1.

Quadro 1 - Padrão de Exatidão Cartográfica - PEC.

\begin{tabular}{|c|c|c|c|c|}
\hline Classe & \multicolumn{2}{|c|}{ Planimetria } & \multicolumn{2}{c|}{ Altimetria } \\
\hline & PEC & EP & PEC & EP \\
\hline A & $0,5 \mathrm{~mm}$ na escala da carta & $0,3 \mathrm{~mm}$ na escala da carta & $1 / 2$ & $1 / 3$ \\
\hline B & $0,8 \mathrm{~mm}$ na escala da carta & $0,5 \mathrm{~mm}$ na escala da carta & $3 / 5$ & $2 / 5$ \\
\hline C & $1 \mathrm{~mm}$ na escala da carta & $0,6 \mathrm{~mm}$ na escala da carta & $3 / 4$ & $1 / 2$ \\
\hline
\end{tabular}

O PEC é um indicador estatístico de dispersão, relativo a $90 \%$ de probabilidade, que define a exatidão de trabalhos cartográficos. A probabilidade de 90\% corresponde a 1,6449 vezes o Erro Padrão (EP, equivalente ao desvio padrão). Nesse contexto, quando se trabalha com a Norma Técnica da Cartografia Nacional, é possível observar a definição do desvio padrão para uma determinada escala, o que possibilita a utilização (alternativa) do desvio padrão "populacional" da classe da carta ao invés de se utilizar o desvio padrão da amostra (NOGUEIRA, 2003).

O teste estatístico para verificação da existência de tendência é então realizado com base na distribuição normal, ou seja:

$$
z=\frac{\Delta \bar{X}}{\alpha_{X}} \cdot \sqrt{n}
$$

e o intervalo de confiança é dado por:

$$
|z|<z_{\alpha / 2}
$$

Logo, se os valores obtidos para a estatística amostral " $z$ " estiverem fora do intervalo de aceitação, rejeita-se a hipótese nula e a carta não pode ser considerada livre de tendência nas coordenadas dos pontos testados, para um dado nível de confiança. 
Segundo Galo e Camargo (1994), a analise de precisão pode ser realizada comparando-se o desvio padrão das discrepâncias com o erro padrão (EP) esperado, para a classe na qual se deseja testar, segundo o PEC. Logo, o teste a ser formulado é apresentado com as seguintes hipóteses:

$\mathrm{H}_{0}: S_{\Delta X}^{2}=\sigma_{X}^{2}$, contra

$\mathrm{H}_{1}: S_{\Delta X}^{2}>\sigma_{X}^{2}$

onde $\sigma_{X}$ corresponde ao desvio padrão esperado para a coordenada $\mathrm{X}$, ou seja, $\mathrm{o}$ erro padão estabelecido segundo a escala e a classe da carta.

Nos trabalhos apresentados por Galo e Camargo (1994), Nogueira (2003) e Galindo (2008) o erro padrão foi fixado não para as coordenadas planimétricas (X e Y) isoladas, mas sim para a resultante, sendo portanto necessário a utilização da seguinte formulação:

$$
\sigma_{X}=\frac{E P}{\sqrt{2}}
$$

Entretanto, de acordo com Nero (2005), o tratamento para a os testes de precisões, utilizando-se variáveis unidimensionais está em concordância com a norma brasileira, que preconiza o $\mathrm{PEC}=1,6449^{*} \mathrm{EP}$ e diz que $90 \%$ dos pontos não devem apresentar erro superior a esse limite. Caso quisesse utilizar o teste na componente planimétrica, a análise seria de uma variável bidimensional e o valor de comparação deveria ser $\mathrm{PEC}=2,146 * \mathrm{EP}$ (coincidente com o CMAS da norma Americana), que corresponde a 90\% (volume abaixo da superfície que representa a probabilidade bidimensional). Dentre várias sugestões para a reformulação da norma brasileira, Nero (2005) comenta sobre a necessidade de deixar claro ao usuário que a análise deve ser efetuada de modo isolado para cada coordenada planimétrica. Nesse caso Equação 7 fica reduzida a:

$$
\sigma_{X}=E P
$$

A etapa seguinte consiste na aplicação do teste Qui-quadrado amostral, dado por:

$$
\chi_{X}^{2}=(n-1) \cdot \frac{S_{\Delta X}^{2}}{\sigma_{X}^{2}}
$$

e, em seguida, verifica se o valor acima calculado está no intervalo de aceitação, ou seja:

$$
\chi_{X}^{2}<\chi_{(n-1, \alpha)}^{2}
$$

Caso a expressão anterior não seja atendida, rejeita-se a hipótese nula, ou seja, o produto cartográfico não atende à precisão estabelecida para " $n-1$ " graus de liberdade a um intervalo de confiança " $\alpha$ ". 


\subsection{Metodologia dos Experimentos}

Os programas necessários para a realização dos experimentos nesta pesquisa, leitura de pontos na imagem, orientação exterior e a intersecção espacial, foram desenvolvidos com base na plataforma computacional MATLAB.

$\mathrm{O}$ processamento e análise dos resultados foram realizados de forma a permitir avaliar o desempenho do modelo matemático da DLT para a orientação exterior do par estereoscópico utilizado nessa pesquisa e também avaliar as exatidões das coordenadas tridimensionais dos pontos de verificação, obtidas via intersecção espacial.

A fase de verificação da adequação do modelo matemático para o propósito dessa pesquisa foi realizada com a utilização da configuração máxima de pontos de apoio. A partir do ajustamento por mínimos quadrados são obtidos os resíduos das observações, matriz variância-covariância dos parâmetros e variância a posteriori, que possibilitam análises sobre a precisão dos parâmetros calculados. Com os parâmetros calculados para o par estereoscópico, a intersecção espacial é realizada para a determinação de coordenadas tridimensionais dos pontos de verificação testados. Com base na comparação dessas coordenadas com as obtidas via levantamento de campo (GPS), as discrepâncias são calculadas. Efetuando-se análises estatísticas dessas discrepâncias junto a valores pré-definidos pelo PEC torna-se possível determinar as exatidões obtidas e concluir sobre a adequação do modelo matemático utilizado nessa aplicação.

Após a realização dos experimentos para analisar a adequação do modelo matemático da DLT aplicado para estereoimagens IKONOS II, a próxima etapa do trabalho tem por objetivo reduzir o número de pontos de apoio utilizados no processo de orientação exterior do par estereoscópico e verificar se as exatidões encontradas para as coordenadas dos pontos de verificação, obtidas via intersecção espacial, são compatíveis com as exatidões obtidas com a configuração máxima de pontos de apoio. Dessa forma, seis configurações de pontos de apoio foram elaboradas, conforme se pode observar na Figura 5. Em todas as configurações de pontos de apoio testadas os parâmetros de orientação exterior do par estereoscópico foram calculados e, a partir desses, procedeu-se ao processo de intersecção espacial para a determinação de coordenadas tridimensionais dos pontos de verificação. Assim como no experimento de adequação do modelo matemático as discrepâncias planialtimétricas são calculadas e testes estatísticos são realizados para analisar os enquadramentos no padrão de exatidão cartográfica, de acordo com a escala requerida.

\section{RESULTADOS E DISCUSSÃO}

Uma das principais vantagens de utilização da transformação matemática direta (DLT) na orientação de estereoimagens orbitais é a não exigência de informações sobre as características internas do sensor, o que permite o relacionamento de forma direta com coordenadas de pontos no sistema de imagem (dado em pixel) com as coordenadas de pontos no terreno. Além disso, seis pontos 
de apoio são suficientes para a determinação dos coeficientes do modelo via ajustamento por mínimos quadrados.

Conforme apresentado na metodologia, foi realizado um experimento com a configuração de 25 pontos de apoio (mostrada na Figura 3) objetivando avaliar o desempenho do modelo matemático testado. Admitiu-se no experimento aqui realizado o valor de um pixel para a precisão das observações, enquanto que as coordenadas de terreno, utilizadas como pontos de apoio, foram admitidas como sendo isentas de erros. Na Tabela 1 são apresentados os resíduos provenientes do ajustamento realizado.

Tabela 1 -Resíduos das observações na imagem 1 e 2.

\begin{tabular}{|c|c|c|c|c|}
\hline \multirow{2}{*}{$\begin{array}{c}\text { código } \\
\text { do ponto }\end{array}$} & \multicolumn{2}{|c|}{ IMAGEM 01} & \multicolumn{2}{|c|}{ IMAGEM 02} \\
\hline & Vx (pixel) & Vy(pixel) & Vx(pixel) & Vy(pixel) \\
\hline 2 & 0,28 & 0,099 & $-0,226$ & 0,311 \\
\hline 9 & 1,165 & 1,067 & 0,815 & $-0,384$ \\
\hline 12 & 0,29 & 0,319 & $-0,211$ & 0,183 \\
\hline 18 & 0,26 & 0,216 & 0,230 & $-0,155$ \\
\hline 23 & 0,149 & 0,067 & 0,047 & $-0,280$ \\
\hline 28 & 0,48 & 0,261 & $-0,147$ & 0,359 \\
\hline 31 & 0,218 & 0,093 & $-0,681$ & 0,228 \\
\hline 34 & $-0,396$ & $-0,472$ & $-0,441$ & 0,531 \\
\hline 35 & 0,167 & 0,030 & $-0,311$ & 0,563 \\
\hline 39 & $-0,845$ & $-0,957$ & 0,237 & $-0,120$ \\
\hline 44 & 0,138 & 0,058 & 0,776 & $-1,030$ \\
\hline 46 & 0,11 & 0,098 & 0,639 & 1,031 \\
\hline 51 & 0,228 & 0,244 & $-0,036$ & $-0,944$ \\
\hline 55 & $-0,106$ & 0,078 & 0,323 & 0,037 \\
\hline 56 & $-0,858$ & $-0,683$ & $-0,390$ & $-0,588$ \\
\hline 61 & $-0,45$ & 0,037 & $-0,177$ & $-0,248$ \\
\hline 64 & 0,009 & 0,107 & $-0,120$ & 0,811 \\
\hline 65 & 0,298 & 0,375 & 1,168 & 0,214 \\
\hline 68 & $-0,166$ & $-0,366$ & $-0,844$ & 0,631 \\
\hline 74 & $-0,139$ & $-0,296$ & $-1,007$ & 0,736 \\
\hline 77 & 0,256 & 0,074 & 0,795 & $-0,067$ \\
\hline 103 & $-0,276$ & $-0,382$ & $-0,163$ & $-0,377$ \\
\hline 106 & 0,186 & 0,323 & 0,211 & $-0,054$ \\
\hline 109 & $-0,616$ & $-0,427$ & 0,057 & $-1,270$ \\
\hline 116 & $-0,384$ & 0,036 & $-0,543$ & $-0,120$ \\
\hline $\begin{array}{l}\text { Desvio- } \\
\text { padrão }\end{array}$ & 0,441 & 0,402 & 0,542 & 0,578 \\
\hline
\end{tabular}


Levando em consideração a precisão pré-definida no processo de ajustamento para as observações e analisando-se os resultados para o desvio padrão da média dos resíduos (TABELA 1) é possível observar que os valores obtidos estão dentro do limite de tolerância. Os resultados dos resíduos comprovam a eficiência do modelo matemático na orientação do par estereoscópico utilizado. A Tabela 2 mostra os resultados dos coeficientes da DLT para o par estereoscópico, o teste do QuiQuadrado entre variâncias a priori com a variância a posteriori e as precisões dos parâmetros calculados.

Tabela 2 - Resultados do ajustamento para 25 pontos de apoio.

\begin{tabular}{c|c|c|c|c}
\hline$\hat{\sigma}_{0}^{2}$ & 0,974 & & & \\
\hline$\chi^{2} v, \frac{\alpha}{2}$ & 125,41 & & $\mathbf{n}^{\mathbf{0}}$ Iterações & \\
\hline$\chi^{2}$ & 149,03 & & 6 & \\
\hline$\chi^{2} v, 1-\frac{\alpha}{2}$ & 182,86 & & gl=153 & \\
\hline & IMAGEM 01 & & IMAGEM 02 & \\
\hline & $\begin{array}{c}\text { Parâm. } \\
\text { ajustados }\end{array}$ & Precisões & $\begin{array}{c}\text { Parûm. } \\
\text { ajustados }\end{array}$ & Precisões \\
\hline POE & 0,881668 & 0,0935299 & 0,8537019 & 0,084366 \\
\hline L1 & $-0,0001909$ & 0,0000796 & $-0,0002401$ & 0,0000712 \\
\hline L2 & 0,1232655 & 0,0113732 & 0,0184228 & 0,0061875 \\
\hline L3 & $-576074,51$ & 61726,46 & $-557312,76$ & 55648,63 \\
\hline L4 & 0,0000186 & 0,0000294 & 0,0001883 & 0,0000324 \\
\hline L5 & $-0,8817804$ & 0,0935091 & $-0,8538129$ & 0,0843466 \\
\hline L6 & $-0,074477$ & 0,010626 & 0,3994317 & 0,0386444 \\
\hline L7 & 6325927,48 & 670841,57 & 6124741,58 & 605054,89 \\
\hline L8 & $-1,57 \mathrm{E}-08$ & $8,37 \mathrm{E}-09$ & $1,19 \mathrm{E}-09$ & $7,92 \mathrm{E}-09$ \\
\hline L9 & $-1,50 \mathrm{E}-08$ & $1,33 \mathrm{E}-08$ & $-2,05 \mathrm{E}-08$ & $1,19 \mathrm{E}-08$ \\
\hline L10 & $-4,59 \mathrm{E}-08$ & $1,69 \mathrm{E}-06$ & $-3,30 \mathrm{E}-07$ & $1,64 \mathrm{E}-06$ \\
\hline L11 & - & & &
\end{tabular}

Apesar da possibilidade de cálculo dos parâmetros físicos do sensor a partir dos coeficientes da DLT para uma imagem de quadro, no experimento aqui realizado tais parâmetros não possuem nenhum relacionamento físico, pois as imagens além de serem geometricamente corrigidas são obtidas a partir de varreduras lineares. Ainda assim é possível, através da MVC, analisar a correlação existente entre parâmetros com o objetivo de obter conclusões sobre as precisões do resultado do ajustamento.

Nas Tabelas 3 e 4 são apresentadas as matrizes com os coeficientes de correlação entre parâmetros para a imagem 1 e 2, respectivamente. Analisando-se os resultados é possível verificar a existência de correlação total entre vários 
parâmetros do modelo matemático. Entretanto, devido ao não significado físico dos parâmetros, torna-se muito difícil a obtenção de causas físicas para essas correlações. Por outro lado, fica evidente que para essa aplicação o modelo DLT encontra-se super parametrizado. Essa particularidade pode também ser vista quando as precisões dos parâmetros são analisadas. Verifica-se que existem aproximadamente três parâmetros sem significado matemático, pois as precisões desses, determinadas na matriz variância e covariância, têm valores maiores ou iguais aos valores determinados pelo ajustamento.

Tabela 3 - Matriz dos coeficientes de correlação entre parâmetros - Imagem 1.

\begin{tabular}{|c|c|c|c|c|c|c|c|c|c|c|c|}
\hline & L1 & L2 & L3 & L4 & L5 & L6 & L7 & L8 & L9 & L10 & L11 \\
\hline L1 & $\mathbf{1}$ & & & & & & & & & & \\
\hline L2 & 0,83 & $\mathbf{1}$ & & & & & & & & & \\
\hline L3 & 0,84 & 0,6 & $\mathbf{1}$ & & & & & & & & \\
\hline L4 & $\mathbf{- 1}$ & $-0,83$ & $-0,84$ & $\mathbf{1}$ & & & & & & & \\
\hline L5 & $-0,04$ & $-0,09$ & 0,05 & 0,04 & $\mathbf{1}$ & & & & & & \\
\hline L6 & $\mathbf{- 1}$ & $-0,83$ & $-0,84$ & $\mathbf{1}$ & 0,04 & $\mathbf{1}$ & & & & & \\
\hline L7 & $-0,86$ & $-0,76$ & $-0,59$ & 0,86 & 0,03 & 0,86 & $\mathbf{1}$ & & & & \\
\hline L8 & $\mathbf{1}$ & 0,83 & 0,84 & $\mathbf{- 1}$ & $-0,04$ & $\mathbf{- 1}$ & $-0,86$ & $\mathbf{1}$ & & & \\
\hline L9 & $-0,21$ & $-0,23$ & $-0,15$ & 0,21 & 0,57 & 0,21 & 0,15 & $-0,21$ & $\mathbf{1}$ & & \\
\hline L10 & $\mathbf{1}$ & 0,83 & 0,83 & $\mathbf{- 1}$ & $-0,07$ & $\mathbf{- 1}$ & $-0,87$ & $\mathbf{1}$ & $-0,26$ & $\mathbf{1}$ & \\
\hline L11 & $-0,38$ & $-0,41$ & 0,03 & 0,38 & $-0,01$ & 0,38 & 0,62 & $-0,38$ & $-0,21$ & $-0,37$ & $\mathbf{1}$ \\
\hline
\end{tabular}

Tabela 4 - Matriz dos coeficientes de correlação entre parâmetros - Imagem 2.

\begin{tabular}{|c|c|c|c|c|c|c|c|c|c|c|c|}
\hline $\mathbf{L 1}$ & $\mathbf{L 1}$ & $\mathbf{L 2}$ & $\mathbf{L 3}$ & $\mathbf{L 4}$ & $\mathbf{L 5}$ & $\mathbf{L 6}$ & $\mathbf{L 7}$ & $\mathbf{L 8}$ & $\mathbf{L 9}$ & $\mathbf{L 1 0}$ & $\mathbf{L 1 1}$ \\
\hline $\mathbf{L 2}$ & $\mathbf{1}$ & & & & & & & & & & \\
\hline L3 & 0,79 & $\mathbf{1}$ & & & & & & & & & \\
\hline $\mathbf{L 4}$ & $-0,24$ & $-0,37$ & $\mathbf{1}$ & & & & & & & & \\
\hline L5 & $\mathbf{- 1}$ & $-0,80$ & 0,24 & $\mathbf{1}$ & & & & & & & \\
\hline L6 & 0,48 & 0,33 & 0,01 & $-0,48$ & $\mathbf{1}$ & & & & & & \\
\hline L7 & $\mathbf{- 1}$ & $-0,79$ & 0,24 & $\mathbf{1}$ & $-0,48$ & $\mathbf{1}$ & & & & & \\
\hline L8 & $\mathbf{0 , 9 9}$ & 0,77 & $-0,17$ & $\mathbf{- 0 , 9 9}$ & 0,48 & $\mathbf{- 0 , 9 9}$ & $\mathbf{1}$ & & & & \\
\hline L9 & $\mathbf{1}$ & 0,79 & $-0,24$ & $\mathbf{- 1}$ & 0,48 & $\mathbf{- 1}$ & $\mathbf{0 , 9 9}$ & $\mathbf{1}$ & & & \\
\hline L10 & 0,00 & $-0,05$ & 0,04 & 0,00 & 0,50 & 0,00 & $-0,01$ & 0,00 & $\mathbf{1}$ & & \\
\hline L11 & $\mathbf{1}$ & 0,80 & $-0,25$ & $\mathbf{- 1}$ & 0,45 & $\mathbf{- 1}$ & $\mathbf{0 , 9 9}$ & $\mathbf{1}$ & $-0,06$ & $\mathbf{1}$ & \\
\hline L1 & $-0,38$ & $-0,41$ & 0,71 & 0,38 & $-0,20$ & 0,38 & $-0,30$ & $-0,38$ & $-0,30$ & $-0,37$ & $\mathbf{1}$ \\
\hline
\end{tabular}

A partir dos coeficientes da função DLT obtidos para o par estereoscópico foi realizado o processo de intersecção espacial para determinação de coordenadas tridimensionais dos pontos de verificação testados. Os valores das discrepâncias obtidos entre as coordenadas calculadas por interseç̧ão espacial e suas respectivas obtidas por levantamento de campo (GPS) são apresentados na Tabela 5. 
Tabela 5 - Discrepâncias entre as coordenadas.

\begin{tabular}{|c|c|c|c|}
\hline \multirow{2}{*}{$\begin{array}{l}\text { id do } \\
\text { ponto }\end{array}$} & \multicolumn{3}{|c|}{ Discrepâncias (m) } \\
\hline & $\mathbf{E}$ & $\mathbf{N}$ & h \\
\hline 7 & $-0,313$ & $-0,644$ & $-1,305$ \\
\hline 15 & $-0,511$ & $-0,384$ & 0,792 \\
\hline 16 & $-0,308$ & $-0,13$ & $-0,917$ \\
\hline 17 & $-0,062$ & 0,346 & $-0,873$ \\
\hline 22 & 0,744 & $-0,752$ & $-0,014$ \\
\hline 24 & 0,37 & $-0,048$ & 0,092 \\
\hline 27 & $-0,178$ & $-0,515$ & $-1,461$ \\
\hline 38 & 0,294 & $-0,245$ & $-0,478$ \\
\hline 54 & 0,849 & 0,967 & $-1,444$ \\
\hline 59 & $-0,272$ & $-0,024$ & 1,732 \\
\hline 60 & $-0,628$ & $-0,678$ & $-1,073$ \\
\hline 62 & 0,909 & 0,34 & $-1,97$ \\
\hline 63 & 0,103 & 0,765 & $-2,987$ \\
\hline 71 & $-0,008$ & 0,383 & 0,747 \\
\hline 75 & $-1,29$ & 0,122 & $-1,213$ \\
\hline 78 & $-0,333$ & $-0,122$ & 0,814 \\
\hline 101 & 0,389 & 0,72 & $-0,657$ \\
\hline 107 & 0,103 & 0,249 & $-1,793$ \\
\hline 111 & 0,418 & 0,985 & $-3,146$ \\
\hline 113 & 0,221 & 0,274 & $-2,635$ \\
\hline media & 0,025 & 0,08 & $-0,889$ \\
\hline $\begin{array}{c}\text { Média } \\
\text { absoluta }\end{array}$ & 0,415 & 0,435 & 1,307 \\
\hline $\begin{array}{l}\text { Desvio } \\
\text { padrão }\end{array}$ & 0,535 & 0,531 & 1,312 \\
\hline
\end{tabular}

Os resultados para o desvio padrão das discrepâncias das coordenadas tridimensionais evidenciam uma distribuição normal, sem presença de tendências e com exatidões com valores compatíveis com as precisões do ajustamento. Esses resultados mostram que apesar da alta correlação entre parâmetros o modelo matemático conseguiu parametrizar as deformações existentes no par estereoscópico. Além disso, a precisão para a componente altimétrica é compatível com a precisão altimétrica esperada, dada pela relação base altura. A representação gráfica das discrepâncias planialtimétrica dos 20 pontos de verificação testados nesse experimento é mostrada na Figura 4. 
Figura 4 - Discrepância planimétrica e altimétrica.

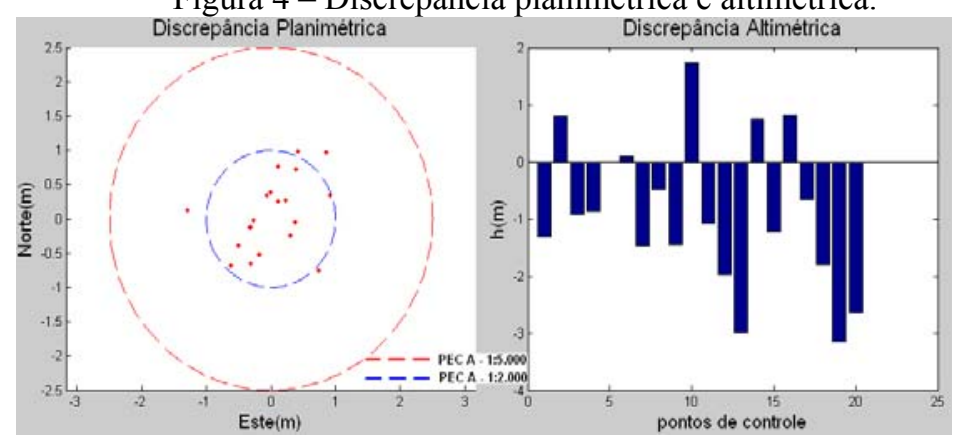

Verificam-se na Figura 4 que as exatidões planimétrica e altimétrica obtidas no experimento realizado encontram-se dentro das exatidões esperadas. Entretanto, objetivando realizar conclusões sobre o enquadramento dos resultados segundo o PEC, foram realizados testes estatísticos de tendência e precisão, e os resultados são apresentados na Tabela 6 .

Tabela 6 - Teste estatístico de tendência e precisão.

\begin{tabular}{c|c|c|c}
\hline \multirow{2}{*}{$\begin{array}{c}\text { Teste } \\
\text { tendência }\end{array}$} & \multicolumn{3}{|c}{ Coordenadas (m) } \\
\cline { 2 - 4 } & $\mathbf{E}$ & $\mathbf{N}$ & $\mathbf{h}$ \\
\hline $\bar{\Delta}(\mathrm{m})$ & 0,025 & 0,080 & $-0,889$ \\
\hline$S_{\Delta X}(\mathrm{~m})$ & 0,535 & 0,531 & 1,312 \\
\hline$Z_{\text {tab }}$ & 1,6449 & 1,6449 & 1,6449 \\
\hline $\mathrm{Z}_{\text {calc }}$ & 0,074 & 0,24 & 2,38 \\
\hline Tendência & $\mathbf{N a ̃ o}$ & $\mathbf{N a ̃ o}$ & $\mathbf{S i m}$ \\
\hline Teste Precisão & & & \\
\hline$\chi_{(1,10 \%)}^{2}$ & 27,2 & 27,2 & \\
\hline$\chi_{\text {ClasseA }}^{2}$ & 2,418 & 2,382 & \\
\hline CLASSE & $\mathbf{A}$ & $\mathbf{A}$ & \\
\hline \multicolumn{4}{|c|}{}
\end{tabular}

Verifica-se na Tabela 6 que a comparação entre o "z" amostral das componentes tridimensionais com o valor teórico $\left(z_{t a b}\right)$ comprova a existência de tendência apenas na componente altimétrica. A possível causa dessa tendência pode estar associada a deformações presentes nas imagens e que não foram parametrizadas pelo modelo matemático aqui testado ou então devido à própria imprecisão da intersecção espacial na determinação da componente altimétrica. Com relação às exatidões resultantes para as componentes planimétricas e suas comprovações via testes estatísticos, tem-se com a aplicação do Qui-Quadrado, o 
enquadramento no PEC planimétrico classe A para uma escala de 1:5.000.

Com a tendência comprovada para a componente altimétrica, o enquadramento dessa componente segundo o PEC foi realizado comparando-se as discrepâncias altimétricas dos pontos de verificação. Na verificação da exatidão altimétrica foi admitido como sendo o PEC em altimetria para escala de 1/5000 com curvas de nível com eqüidistância de 5 metros, o valor de 1,6449 vezes o erro padrão ou $90 \%$ dos pontos testados não deverão apresentar erros superiores ao PEC altimétrico estabelecido. Nessa condição, os erros padrões para classe A, B e C são $(1,667),(2,0)$ e $(2,5)$ metros respectivamente, resultando para o PEC altimétrico os valores de $(2,74),(3,29)$ e $(4,16)$ metros, respectivamente para as classes A, B e C. Com base nesses valores, e analisando-se as discrepâncias altimétricas (Tabela 5) dos 20 pontos de verificação é possível enquadrar os resultados altimétricos como sendo da classe A.

A partir da configuração inicial de (25) pontos de apoio, diversos experimentos foram realizados com diferentes configurações de pontos de apoio no modelo estereoscópico. O objetivo principal dos experimentos realizados com diferentes configurações de pontos de apoio foi o de obter a menor quantidade de pontos de apoio e sua distribuição espacial que permitisse obter exatidões planimétricas e altimétricas equivalentes com a obtida na configuração inicial com elevado número de pontos de apoio. Pode-se observar na Figura 5 que os experimentos a seguir foram realizados com 20,12, 9 e seis pontos de apoio. Observa-se ainda na Figura 5 que duas configurações são propostas para as quantidades de 12 e 9 pontos de apoio, sendo uma delas com pontos localizados apenas nas extremidades da cena.

Figura 5 - Configurações com pontos distribuídos.

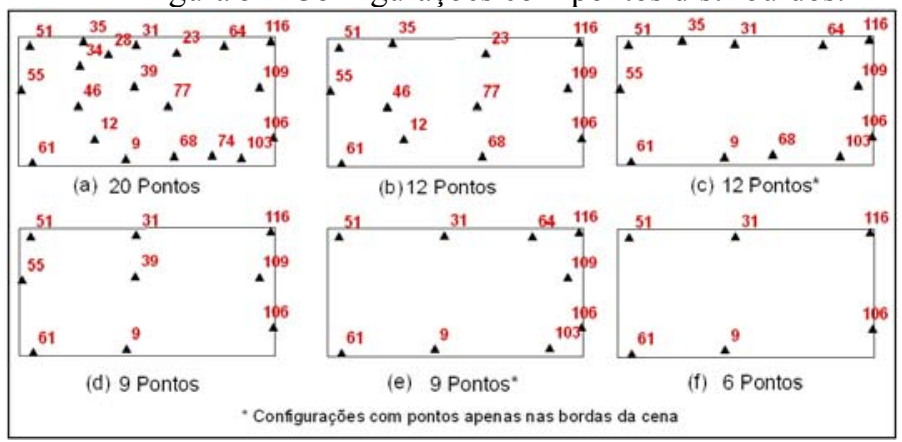

Os experimentos aqui realizados consistem do ajustamento por mínimos quadrados para a determinação dos coeficientes do modelo DLT para o par estereoscópico, a intersecção espacial nos pontos de verificação e as análises estatísticas das discrepâncias para o enquadramento das exatidões planimétricas e altimétricas no PEC para a escala de 1:5.000. 
A Tabela 7 mostra os resultados que foram obtidos das analises estatísticas realizadas com os valores das discrepâncias planialtimétricas dos vinte pontos de verificação com as diferentes configurações de pontos de apoio apresentadas na Figura 6.

Tabela 7 - Resultados obtidos da análise das discrepâncias.

\begin{tabular}{|c|c|c|c|c|c|c|c|c|c|}
\hline Config. & Coord. & $\bar{\Delta}(\mathrm{m})$ & $\sigma(\mathrm{m})$ & $\mathrm{z}_{\text {calc }}$ & $\mathbf{T}$ & $\chi_{A}^{2}$ & PEC & \multicolumn{2}{|c|}{ RMSE (m) } \\
\hline & Este & $-0,04$ & 0,51 & 0,13 & $\mathrm{~N}$ & 2,21 & $\mathrm{~A}$ & Este & 0,50 \\
\hline \multirow[t]{3}{*}{20 pts } & Norte & 0,02 & 0,55 & 0,06 & $\mathrm{~N}$ & 2,52 & $\mathrm{~A}$ & Norte & 0,53 \\
\hline & h & $-0,86$ & 1,37 & 2,30 & $\mathrm{~S}$ & & A & $\mathrm{h}$ & 1,59 \\
\hline & Este & 0,02 & 0,55 & 0,06 & $\mathrm{~N}$ & 2,56 & A & Este & 0,53 \\
\hline \multirow[t]{3}{*}{12 pts } & Norte & 0,09 & 0,49 & 0,27 & $\mathrm{~N}$ & 1,99 & $\mathrm{~A}$ & Norte & 0,48 \\
\hline & h & $-0,87$ & 1,72 & 2,34 & S & & A & $\mathrm{h}$ & 1,88 \\
\hline & Este & 0,02 & 0,50 & 0,07 & $\mathrm{~N}$ & 2,10 & $\mathrm{~A}$ & Este & 0,48 \\
\hline \multirow[t]{3}{*}{ 12* pts } & Norte & $-0,11$ & 0,53 & 0,33 & $\mathrm{~N}$ & 2,33 & $\mathrm{~A}$ & Norte & 0,52 \\
\hline & h & $-1,34$ & 1,40 & 3,60 & S & & B & $\mathrm{h}$ & 1,91 \\
\hline & Este & 0,30 & 0,47 & 0,90 & $\mathrm{~N}$ & 1,87 & $\mathrm{~A}$ & Este & 0,55 \\
\hline \multirow[t]{3}{*}{9 pts } & Norte & $-0,07$ & 0,59 & 0,21 & $\mathrm{~N}$ & 2,90 & $\mathrm{~A}$ & Norte & 0,57 \\
\hline & h & $-1,95$ & 1,41 & 5,24 & $\mathrm{~S}$ & & C & $\mathrm{h}$ & 2,38 \\
\hline & Este & 0,18 & 0,44 & 0,54 & $\mathrm{~N}$ & 1,64 & A & Este & 0,46 \\
\hline \multirow[t]{3}{*}{ 9* pts } & Norte & $-0,14$ & 0,52 & 0,43 & $\mathrm{~N}$ & 2,31 & A & Norte & 0,53 \\
\hline & h & $-1,83$ & 1,45 & 4,91 & S & & C & $\mathrm{h}$ & 2,31 \\
\hline & Este & 0,26 & 0,43 & 0,76 & $\mathrm{~N}$ & 1,55 & A & Este & 0,49 \\
\hline \multirow[t]{2}{*}{6 pts } & Norte & 0,00 & 0,77 & 0,01 & $\mathrm{~N}$ & 4,95 & $\mathrm{~A}$ & Norte & 0,74 \\
\hline & h & $-1,59$ & 1,39 & 4,27 & S & & B & $\mathrm{h}$ & 2,08 \\
\hline
\end{tabular}

Analisando-se os resultados apresentados na Tabela 7 verifica-se que todas as exatidões planimétricas obtidas foram enquadradas, com base no teste estatístico do Qui-Quadrado com nível de $10 \%$ de significância, no PEC planimétrico para a escala de $1 / 5000$ - classe A. Com relação à exatidão altimétrica, a pequena tendência na determinação dessa componente foi mantida, como ocorreu no experimento com 25 pontos de apoio. O comportamento da tendência altimétrica é função da configuração dos pontos de apoio, como pode ser observado na Figura 6.

Verifica-se na Figura 7 que o valor do erro médio quadrático na altimetria, em cada uma das configurações de pontos de apoio, reflete a tendência altimétrica existente. Considerando os valores de desvios padrão apresentados na Tabela 7 , verifica-se que independente da configuração de pontos de apoio utilizada, a determinação da componente altimétrica sempre foi realizada com mesma precisão, mas com diferentes exatidões. 
Figura 6 - Média das discrepâncias planialtimétricas.

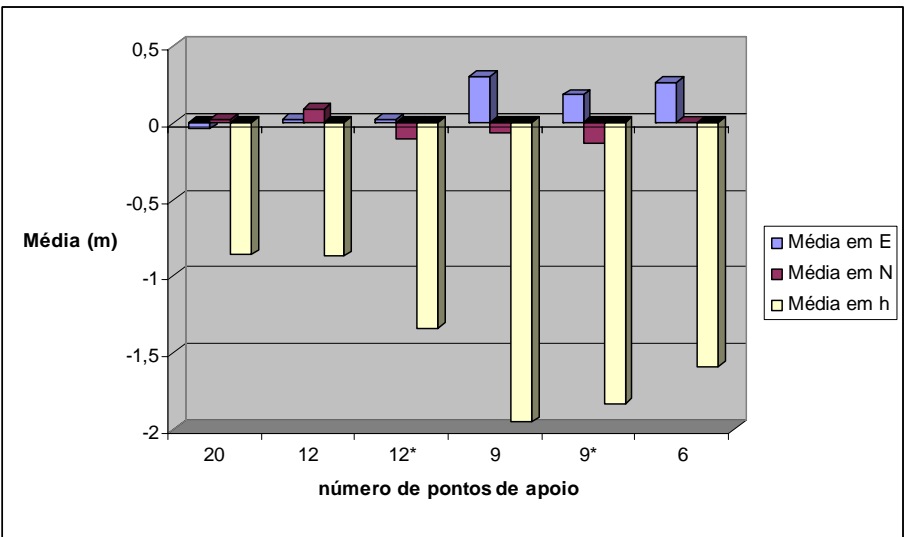

Figura 7 - Erro médio quadrático das discrepâncias planialtimétricas.

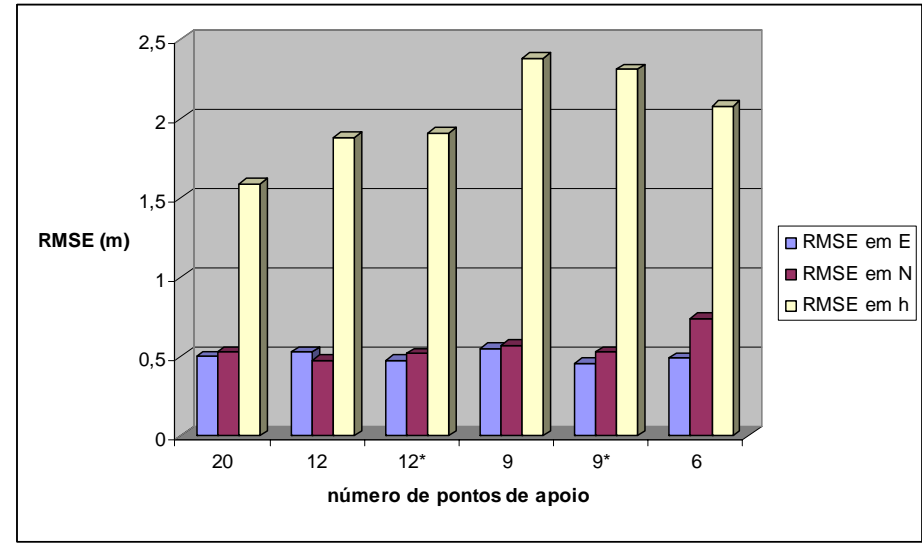

Com respeito à tendência sempre existente na determinação altimétrica podese inferir a grande possibilidade da existência de um erro sistemático não parametrizado nos parâmetros RPC responsável pela geração das imagens do par estereoscópico. Entretanto, os resultados obtidos de exatidão altimétrica nos experimentos realizados podem ser considerados como sendo muito bons, considerando o valor da precisão esperada na determinação da componente altimétrica via intersecção espacial, dada pela seguinte expressão (KRAUSS, 1993):

$$
d z= \pm \frac{H^{2}}{B \cdot f} \cdot d_{p x}
$$


Para essa equação, $d z$ é a precisão esperada para a componente altimétrica, $H$ é a altura de vôo, $B$ a base fotogramétrica, $f$ a distância focal e $d_{p x}$ é a precisão com que as coordenadas de imagem dos pontos homólogos foram obtidas. Adotando-se os dados do par estereoscópico utilizados $(H=680 \mathrm{~km}, B=340 \mathrm{~km}, f=10 \mathrm{~m}$ e para $d_{p x}=12 \mu \mathrm{m}$ ) resulta um $d z$ de 1,6 metros.

Analisando-se de forma individual o comportamento da DLT com as configurações de pontos de apoio testadas, admite-se a configuração (b) com 12 pontos de apoio como sendo a mais apropriada para a determinação altimétrica e no caso de aplicação exclusiva para a determinação planimétrica a configuração (f) com seis pontos de apoio pode ser considerada como a mais indicada.

\section{CONCLUSÕES E RECOMENDAÇÕES}

O presente trabalho teve como principal objetivo avaliar o desempenho do modelo matemático da DLT na geração de coordenadas tridimensionais via intersecção espacial e também uma configuração ótima de pontos de apoio que possibilite a determinação precisa das coordenadas tridimensionais. Para tanto foi tomado como aproximação que as imagens orbitais foram obtidas de forma instantânea ou provenientes de um sensor de quadro. Os experimentos realizados permitem apresentar as seguintes conclusões:

De forma geral, os experimentos realizados demonstraram que é possível empregar o modelo matemático da DLT nas estereoimagens IKONOS II utilizadas nessa pesquisa para a geração de coordenadas planialtimétricas;

$\mathrm{Na}$ determinação das componentes planimétricas foi possível observar que os resultados obtidos apresentaram exatidões que se enquadram no PEC planimétrico classe A para a escala de 1:5.000. Já para a determinação da componente altimétrica foi possível constatar uma pequena tendência para todas as configurações de pontos de apoio testadas e, apenas as configurações (a) e (b) puderam ser enquadradas no PEC altimétrico classe A. Apesar dessa tendência verificada na determinação das coordenadas altimétricas e considerando-se o valor de precisão esperada para a coordenada altimétrica os resultados obtidos para a coordenada altimétrica podem ser considerados como sendo satisfatórios.

Analisando-se de forma geral o comportamento da DLT com as configurações de pontos de apoio testadas, admiti-se a configuração (b) com 12 pontos de apoio como sendo a mais apropriada para a determinação altimétrica e no caso de aplicação exclusiva para a determinação planimétrica a configuração (f) com seis pontos de apoio pode ser considerada como a mais indicada.

Com relação à utilização do modelo matemático da DLT em atividades práticas deve-se ressaltar a importância de se obter coordenadas de imagem e de terreno livre de erros grosseiros. $\mathrm{Na}$ aplicação em questão, pode-se afirmar que o modelo teve um ótimo desempenho. Entretanto a sua utilização em procedimentos práticos dessa natureza pode ser questionada em função da forte correlação existente entre parâmetros o que evidencia a ocorrência de modelo super-parametrizado. Essa condição pode trazer instabilidade na resolução do sistema de equações como 
também a grande possibilidade de absorção de erros grosseiros, falseando as precisões obtidas no ajustamento e acarretando inexatidões no processo.

\section{AGRADECIMENTOS}

Os autores agradecem ao Curso de Pós-Graduação em Ciências Geodésicas CPGCG/UFPR, à agência de fomento CAPES, pela concessão da bolsa de estudos e incentivo à pesquisa e ao $\mathrm{CNPq}$ pela concessão de recursos financeiros para aquisição do par estereoscópico de imagens de alta resolução Ikonos II (Projeto 477222/2003-0).

\section{REFERÊNCIAS BIBLIOGRÁFICAS}

ABDEL-AZIZ, Y.I.; KARARA, H. M. Direct Linear Transform from Comparator Coordinates into Object Space Coordinates in Close-Range. Photogrammetry, Proceedings of the AUI/UI Symposium on Close-Range Photogrammetry, Urbana, v.1, n.18, 1971.

AMATO, R.; DARDANELLI, G.; EMMOLO, D.; FRANCO, V.; LO BRUTTO, M.; MIDULLA, P.; ORLANDO, P.; VILLA, B. Digital Orthophotos at scale of 1:5000 from high resolution satellite imagens. ISPRS Journal of Photogrammetry \& Remote Sensing. Vol XXXV, part B5, p. 593-598. 2004.

BRASIL. Decreto $\mathrm{n}^{\circ}$. 89.817, de 20 de junho de 1984. Dispõe sobre as instruções reguladoras das normas técnicas da cartografia nacional. Diário Oficial da República Federativa do Brasil, Brasília, 22 de junho de 1984.

DAL POZ, A. P.; SCALCO, J. C. Orientação semi-automática de imagens CBERS usando rodovias como controle de campo. Boletim de Ciências Geodésicas, v.12, n.2, p.295-313, Curitiba, 2006.

DAL POZ, A. P.; SCALCO, J.C.; Orientação Semi-Automática de Imagens CBERS Usando Rodovias como Controle de Campo. Boletim de Ciências Geodésicas, Curitiba,v.12, n.2, p.295-313, jul.-dez. 2006.

EL-MANADILI, Y.; NOVAK, K. Precision Rectification of SPOT Imagery using the Direct Linear Transformation Model. Photogrammetric Engineering and Remote Sensing, v. 62, 1996. p. 67-72.

FRASER, C. S.; HANLEY, H. B.; YAMAKAWA, T. Three-Dimensional Geopositioning Accuracy of IKONOS imagery sub-metre geopositioning with IKONOS geo imagery. Photogrammetric Record, v. 17, n.99, p. 465-479, 2002.

FRITSCH, D.; STALLMANN, D. Rigorous Photogrammetric Processing Of High Resolution Satellite Imagery. ISPRS Journal of Photogrammetry \& Remote Sensing, Vol XXXIII 2000.

GALINDO, J. R. F. Análise da reconstrução 3D a partir de um par estereoscópico HR-CCD/CBERS-2 usando dois modelos matemáticos. Presidente Prudente: UNESP, 2008. 107 f. Dissertação (Mestrado em Ciências Cartográficas) Programa de Pós-Graduação em Ciências Cartográficas, Faculdade de Ciência e Tecnologia, Universidade Estadual Paulista, Presidente Prudente, 2008. 
GALO, M.; CAMARGO, P. O. Utilização do GPS no controle de qualidade de cartas. In: Congresso Brasileiro de Cadastro Técnico Multifinalitário COBRAC, 1994, Florianópolis. Anais... Florianópolis SC, 1994, p..41-48.

GRODECKI, J. Ikonos Stereo feature extraction - RPC approach. In ASPRS Annual Conference, 2001, Proceedings ..., St. Louis, 23-27 Abril, 2001.

JACOBSEN, K.; Comparision of Image Orientation by Ikonos, Quickbird and Orbview-3, New Developments and Challenges in Remote Sensing, Z. Bochenek Millpress, Rotterdam, p. 667-676, 2007

JUBANSKI, J. J.; MITISHITA, E. A. Avaliação da Determinação de Coordenadas Tridimensionais Extraídas de um Par Estereoscópico “EROS A”, Utilizando-se da "Direct Linear Transformation” (DLT). Simpósio de Ciências Geodésicas e Tecnologias da Informação, 2004, Recife, 2004.

KIM, T.; DOWMAN, I. Comparision of two Physical Sensor Models for Satellite Images: Position-Rotation model and Orbit-Attitude model. The Photogrammetric Record, v.21, n 114, p. 110-123, 2006.

KRAUS, K. Photogrammetry - Fundamentals and Standard Process.UMLLER/ BONN, v.1, 1993.

LUGNANI, J.B . Introdução à Fototriangulação. Curitiba: Ed UFPR, 1987. 134p.

MERCHANT, D.C. Analytical Photogrammetry: Theory and Practice. Columbus, Ohio, 1979, Department of Geodetic Science, The Ohio state University.

MERCHANT, D.C. Spatial Accuracy Standards for Large Scalle Line Maps. In: Technical Congress on Surveing and Mapping. Proceedings. v. 1, p. 222-231, 1982.

MITISHITA, E. A.; SARAIVA, C. C. S; Machado, A. M. Monorrestituição de Imagens de Alta Resolução Ikonos 2 (GEO), Utilizando-se da Transformação DLT e Modelo Digital do Terreno. XI Simpósio Brasileiro de Sensoriamento Remoto, Belo Horizonte:2003. Anais do Congresso, CD-Rom, Belo Horizonte, 2003.

NERO, M. A. Propostas para o controle de qualidade de bases cartográficas com ênfase na componente posicional. TESE (Doutorado em Engenharia) - Escola Politécnica da Universidade de São Paulo, São Paulo, 2005, 186p.

NOGUEIRA JR.,J. B. Controle de qualidade de produtos cartográficos: uma proposta metodológica. Dissertação de mestrado, Universidade Estadual Paulista, Faculdades de Ciência e Tecnologia de Presidente Prudente/SP, 2003. $143 \mathrm{p}$.

PEDRO, P. C. Ortorretificação de Imagens de Alta Resolução Ikonos e QuickBird Utilizando o Modelo Afim (Affine Projection Model). Curitiba: UFPR, 2005. Dissertação (Mestrado em Ciências Geodésicas) - Curso de Pós-Graduação em Ciências Geodésicas, Universidade Federal do Paraná, Curitiba, 2005.

SAVOPOL, F.; ARMENAKIS, C. Modelling of the IRS-1C Satellite Pan StereoImagery using DLT approach. International archives of Photogrammetry and Remote Sensing.v. 32, part 4, CommIV, p. 511-514. 1998. 
YAMAKAWA, T.; FRASER, C. S. The Affine Projection Model for Sensor Orientation: Experiences With High-Resolution Satellite Imagery. ISPRS Journal of Photogrammetry \& Remote Sensing. Vol XXXV, part B1, p. 142147. 2004.

WOLF, P. R. Elements of Photogrammetry, 3 ed. New York: McGraw-Hill, 2004. $628 \mathrm{p}$.

(Recebido em dezembro de 2009. Aceito em janeiro de 2012). 